\title{
Validation of a single-channel airflow monitor for screening of sleep-disordered
} breathing

\author{
H. Nakano*, T. Tanigawa\#", Y. Ohnishi", H. Uemori*, K. Senzaki", \\ T. Furukawa* and S. Nishima*
}

ABSTRACT: A simple screening method for sleep-disordered breathing (SDB) is desirable for primary care practices. In the present study, a simple monitor, which utilises a new type of flow sensor and a novel algorithm, was prospectively validated.

Home recording for 2 nights with the monitor only, followed by in-laboratory recording with the monitor together with polysomnography, were carried out in consecutive patients $(n=100)$ suspected of SDB. A subjective sleep log was also recorded. The signal was analysed using power spectral analysis, which yielded the flow respiratory disturbance index (flow-RDI).

There was no recording failure at home. The reproducibility of the flow-RDI between the 2 nights at home was high (intraclass correlation coefficient $=0.92$ ). The sensitivity and specificity of the in-laboratory flow-RDI to diagnose SDB were 0.96 and $0.82,0.91$ and 0.82 , and 0.89 and 0.96 , for apnoea/hypopnoea index $(\mathrm{AHI}) \geqslant 5, \geqslant 15$ and $\geqslant 30$ events $\cdot \mathrm{h}^{-1}$, respectively. The diagnostic ability in low-severity subgroups (female, normal weight, AHI $<15$ events $h^{-1}$ ) was almost comparable to that in the entire group. Excluding subjective waking time on the sleep log from the recording time had no significant effect on the flow-RDI.

The single-channel monitor is considered feasible for ambulatory sleep disordered breathing monitoring because of its easy applicability, high reproducibility and relatively high agreement with polysomnography results.

KEYWORDS: Power spectral analysis, screening, sleep apnoea, thermal sensor

leep-disordered breathing (SDB) is recognised as being a very prevalent disease [1], which causes excessive daytime sleepiness resulting in impaired quality of life and an increased risk of motor vehicle crashes. Moreover, SDB is considered to be an independent risk factor for cardiovascular disease [2]. Therefore, it would be desirable to perform a simple screening test for SDB in primary care practices for suspected patients or in health check-ups for the general population. Ordinary polysomnography (PSG) is a very time-consuming and labour-intensive process, and it may not be applicable for this purpose. Although many kinds of portable monitors for SDB have been developed, the American Academy of Sleep Medicine has recommended only a "type 3 " device under both the in-laboratory and attended conditions as an acceptable modality [3]. The type 3 device incorporates at least 4 channels, including two for respiratory movement and/or airflow, one for ECG or heart rate, and one for oxygen saturation. One of the most important problems for the ambulatory use of a type 3 monitor is data loss due to recording failure. For example, reported failure rates for type 3 home monitoring have been variously reported as 5.8, 10 and $18 \%$ [4-6]. The set-up procedure for a type 3 device does not appear to be easy for many subjects. One study compared the rate of recording failure between subjects with and without a sleep technician's set-up at home (7 versus 33\%), indicating the necessity of the technician's assistance with the set-up [7]. A simpler method is desirable for SDB screening in primary care practices. Recently, several single-channel airflow monitors have been developed for automated detection of SDB [8-10]. These devices seem suitable for such a purpose. However, studies on the accuracy of these monitors have provided conflicting results. Both analytical algorithms and flow sensors are crucial parts of such monitors. In this context, the current authors have developed an algorithm to detect SDB from a single-channel airflow record [11]. The algorithm was tested using retrospective samples of airflow records
AFFILIATIONS

*Dept of Pulmonology, Fukuoka National Hospital, Fukuoka, \#Dept of Public Health, Graduate School of Medicine, Ehime University, Ehime, and 'Dept of Internal Medicine, Tenri City Hospital, Tenri, Japan.

CORRESPONDENCE

H. Nakano

Dept of Pulmonology

Fukuoka National Hospital

4-39-1 Yakatabaru

Minami-ku

Fukuoka

811-1394

Japan

Fax: 81925669444

E-mail: nakano h@

palette.plala.or.jp

Received:

October 042007

Accepted after revision:

April 262008

STATEMENT OF INTEREST

Statements of interest

for H. Nakano and T. Tanigawa, and the study itself can be found at www.erj.ersjournals.com/misc/ statements.shtml 
from PSG and has been demonstrated to be relatively accurate and immune to noise contamination. However, validation of the algorithm in a real-life situation has not been performed to date. The primary aim of the present study is to evaluate prospectively the performance of a single-channel airflow monitor, which utilises a new type of flow sensor, combined with the analytical algorithm. An inevitable weakness of such a monitor is the inability to calculate real sleep time. The current authors speculated that a subjective sleep log could help to overcome such a weakness; therefore, an additional aim is to evaluate the usefulness of a subjective sleep $\log$

\section{METHODS}

\section{Subjects}

This study was conducted in two hospitals: Fukuoka National Hospital (FNH; Fukuoka, Japan); and the Tenri City Hospital (TCH; Tenri, Japan). In each hospital, 50 consecutive patients who met the inclusion criteria were invited to participate in this study. Inclusion criteria were patients referred for suspected sleep apnoea, who were eligible to undergo diagnostic PSG and whose age was $\geqslant 18$ and $<65$ yrs. The study protocol was approved by the ethics committees of $\mathrm{FNH}$ and $\mathrm{TCH}$, and all participants gave written informed consent prior to the study.

\section{Study protocol}

The study consisted of recordings made consecutively on 3 nights. During the first and second nights, home recording was carried out with the portable monitor alone. During the third night, simultaneous recording with ordinary PSG and the portable monitor was performed in the hospital. The subjects were asked to note subjective sleep time on a sleep log just after awaking in the morning (FNH: for all 3 nights; TCH: for the 2 nights at home). The subjects received the portable monitor at the first visit to the hospital, when a hospital technician made a brief ( $\sim 5 \mathrm{~min}$ ) instruction about the method for attaching the sensor to their face and operation of the device. An instruction leaflet with the sleep log was also provided for the subjects.

\section{Portable monitor}

A single-channel airflow monitor (SOMNIE; NGK Spark Plug Co. Ltd, Nagoya, Japan) was used as the portable monitor (fig. 1). The SOMNIE utilises a polyvinylidene fluoride (PVDF) film as the thermal sensor to detect airflow. The device stores the airflow signal as digital data at a sampling frequency of $10 \mathrm{~Hz}$ and has the capacity to record data for $24 \mathrm{~h}$. The airflow data can be reviewed if necessary (fig. 2). The airflow sensor is designed to detect both nasal and oral breathing.

\section{PSG}

PSG was recorded using a polygraph system (TCH: MME-3116; FNH: EEG7414; Nihon Kohden, Tokyo, Japan). Nasal airflow was monitored with a nasal prong pressure transducer (PTAF; Pro-Tec, Mukilteo, WA, USA). Nasal prongs were modified to be inserted into the nostrils so as not to interfere with the SOMNIE sensor attached below the nose. Thoracic and abdominal respiratory movements were monitored with respiratory inductive plethysmography (RIP; Respitrace; Ambulatory Monitoring Inc., Ardsley, NY, USA). Oxyhaemoglobin saturation was monitored using a pulse oximeter (OLV-3100; Nihon Kohden,
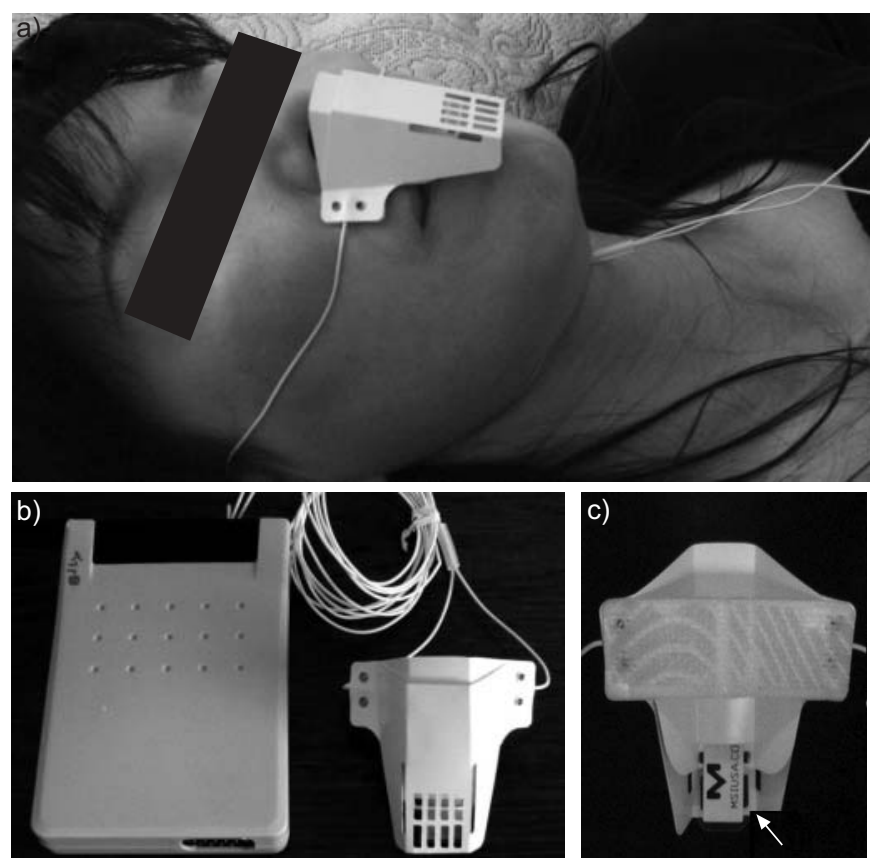

FIGURE 1. The single-channel monitor SOMNIE. a) The SOMNIE disposable sensor (NGK Spark Plug Co. Ltd, Nagoya, Japan) attached below the nose. b) The recording device. c) The back of the sensor. The arrow indicates the polyvinylidene fluoride sensor.

Tokyo, Japan) at the fastest response mode. The recording of the PSG and that of the SOMNIE were started simultaneously.

Sleep stages were scored manually according to standard criteria [12]. The oxygen desaturation index (ODI) was defined by the number of arterial oxygen saturation dips $(\geqslant 3 \%)$ per hour of examination. Apnoea was defined as an episode of clear airflow amplitude reduction $(\geqslant 50 \%)$ lasting $>10 \mathrm{~s}$, while hypopnoea was defined as an episode of discernible airflow amplitude reduction $(<50 \%)$ lasting $>10 \mathrm{~s}$ associated with a $\geqslant 3 \%$ oxygen desaturation or an arousal, according to the research definition of the American Academy of Sleep Medicine $[13,14]$. The detection of apnoea and hypopnoea primarily employed airflow signal obtained by square root transformation of the nasal prong pressure. When the nasal pressure signal became inadequate (e.g. through oral breathing), RIP sum signal amplitude reduction was used as the substitution. The scorers were blinded to the result of the SOMNIE. The apnoea/ hypopnoea index (AHI) was calculated as the number of apnoea and hypopnoea events per hour of sleep.

\section{Analysis}

The data from the SOMNIE were analysed automatically using a computer program (Flow.exe; Institute of Sleep Health Promotion, Tsukuba, Japan). This program utilises short-time power spectral analysis and yields an index, the flow respiratory disturbance index (flow-RDI). The algorithm has been described in detail previously [11]. It is known that there is a systematic bias between the flow-RDI and AHI which correlates to the AHI. Therefore, a cut-off value was used to diagnose SDB, which was determined from a regression analysis between the flow-RDI and AHI in a retrospective sample of PSG records, including PVDF thermal flow sensor 


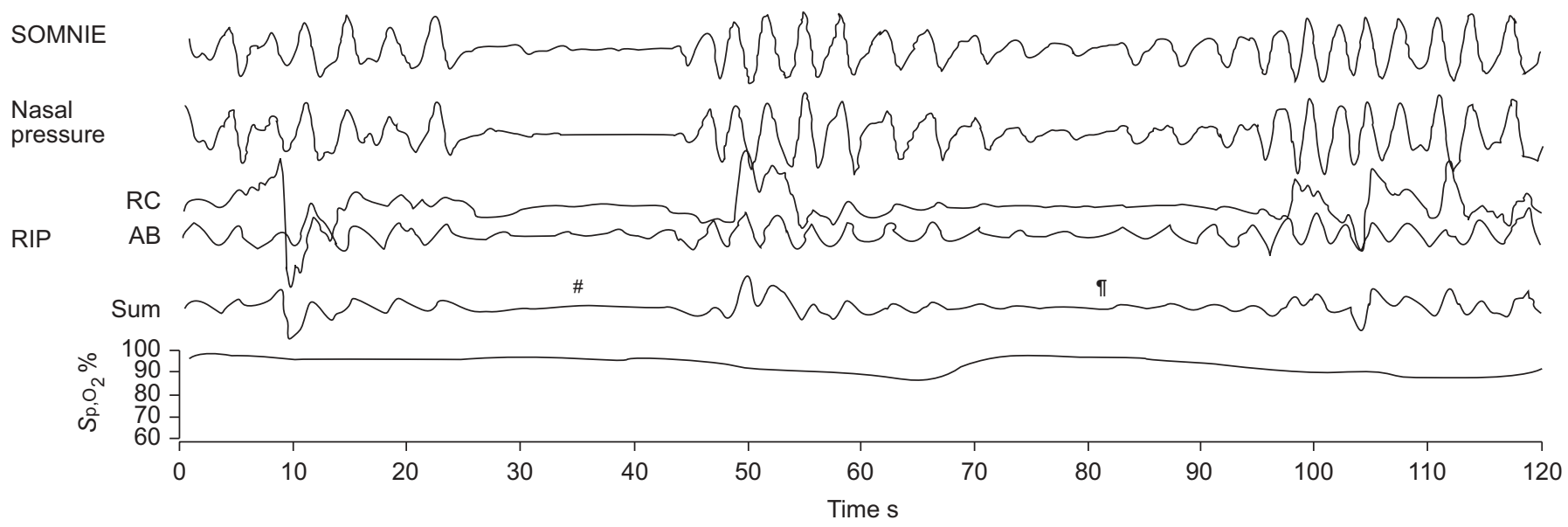

FIGURE 2. Example of respiratory signal waveforms from polysomnography and simultaneous SOMNIE (NGK Spark Plug Co. Ltd, Nagoya, Japan) record. The nasal pressure was square root transformed. One apnoea $\left(^{\#}\right)$ and one hypopnoea (") event is shown. Sp, $\mathrm{O}_{2}$ : arterial oxygen saturation measured by pulse oximetry; RIP: respiratory inductive plethysmography (Respitrace, Ambulatory Monitoring Inc., Ardsley, NY, USA); RC: rib cage; AB: abdomen.

records. The cut-off value was $5.3,11.4$ and 19.6 events $\cdot h^{-1}$ for the SDBs of AHIs $\geqslant 5,15$ and 30 events $\cdot h^{-1}$, respectively.

Reproducibility of the flow-RDI was evaluated using an intraclass correlation coefficient among the data from the 3 nights.

The relationship between the flow-RDI and the AHI was evaluated using Pearson's correlation coefficient. The agreement between these data was analysed as described by BLAND and ALtMAN [15]. The diagnostic ability of the flow-RDI for SDB was evaluated in terms of sensitivity and specificity. All the analyses were performed for each hospital separately and for the entire combined sample. The same analyses were performed for the ODI versus AHI relationship, and the relationship was compared between normal weight subjects (body mass index (BMI) $<25 \mathrm{~kg} \cdot \mathrm{m}^{-2}$ ) and overweight subjects $\left(B M I \geqslant 25 \mathrm{~kg} \cdot \mathrm{m}^{-2}\right)$. The diagnostic ability of flow-RDI was also evaluated for three subgroups of female subjects, normal weight subjects (BMI $<25 \mathrm{~kg} \cdot \mathrm{m}^{-2}$ ) and subjects with normal or mild SDB (AHI $<15$ events $\cdot h^{-1}$ ).

In addition, in order to determine whether a subjective sleep log is useful for improving diagnostic accuracy of the portable monitor, the same analysis was performed for the SOMNIE home night 1 data $(n=25)$ and hospital night data $(n=16)$ after excluding the subjective waking segment of the sleep diary in subjects whose subjective sleep time was $<80 \%$ of recording time.

To evaluate event-by-event agreement, comparison was made between the events detected by three methods of automated detection by the SOMNIE, visual inspection of the SOMNIE airflow record, and that of full PSG records, during $1 \mathrm{~h}$ of mid sleep in all cases. The event-by-event agreement was evaluated using the proportion of specific agreement (PSA) [16].

As an estimate of the clinical utility of the portable monitor, the relationship between the SDB severity measured by SOMNIE's home night 1 data and continuous positive airway pressure (CPAP) use at $1 \mathrm{yr}$ after the PSG was investigated. According to the Japanese medical insurance system, CPAP therapy was prescribed for the patients with AHI of $\geqslant 20$ events $\cdot h^{-1}$ when they accepted the treatment. The data of the home monitoring were not taken into account for the decision of treatment.

\section{RESULTS}

Patient characteristics are shown in table 1. Their presenting symptoms included habitual snoring $(n=80)$, daytime sleepiness $(n=64)$ and witnessed apnoea $(n=63)$. There was no recording failure at all in either the home or in-laboratory studies. Their final diagnoses were obstructive sleep apnoea hypopnoea syndrome $(n=83)$, primary snoring $(n=12)$, insufficient sleep $(n=4)$ and depression $(n=1)$. No patients had significant periodic leg movement disorder.

\section{TABLE 1 Subject characteristics}

\begin{tabular}{|c|c|c|}
\hline & FNH & $\mathrm{TCH}$ \\
\hline Subjects $n$ & 50 & 50 \\
\hline Male/female $n$ & $40 / 10$ & $40 / 10$ \\
\hline Age yrs & $44.2 \pm 10.6$ & $46.3 \pm 10.7$ \\
\hline Body mass index $\mathrm{kg} \cdot \mathrm{m}^{-2}$ & $26.4 \pm 4.1$ & $27.4 \pm 4.4$ \\
\hline AHI events $\cdot h^{-1}$ & $27.4(9.5-47.6)$ & $33.6(12.3-65.2)$ \\
\hline ODI events $\cdot h^{-1}$ & $15.8(5.8-36.4)$ & $25.8(8.3-41.3)$ \\
\hline Sleep efficiency \% & $80.9 \pm 10.7$ & $76.3 \pm 11.9$ \\
\hline \multicolumn{3}{|l|}{ Patients with AHI n } \\
\hline$\geqslant 5$ events $\cdot h^{-1}$ & $44(88)$ & $45(90)$ \\
\hline$\geqslant 15$ events $\cdot h^{-1}$ & $30(60)$ & $35(70)$ \\
\hline$\geqslant 30$ events $\cdot h^{-1}$ & $24(48)$ & $28(56)$ \\
\hline Flow-RDI home night 1 events $\cdot h^{-1}$ & $14.2(8.5-32.9)$ & $16.3(8.4-31.9)$ \\
\hline Flow-RDI home night 2 events $\cdot h^{-1}$ & $15.1(8.6-37.5)$ & $15.7(7.8-36.1)$ \\
\hline Flow-RDI hospital night events $\cdot h^{-1}$ & $16.5(8.8-36.0)$ & $22.9(10.3-45.1)$ \\
\hline
\end{tabular}

Data are presented as mean $\pm \mathrm{SD}$, median (interquartile range), or $\mathrm{n}(\%)$. $\mathrm{FNH}$ : Fukuoka National Hospital, Fukuoka, Japan; TCH: Tenri City Hospital, Tenri, Japan; AHI: apnoea/hypopnoea index; ODI: oxygen desaturation index at the threshold of $3 \%$; flow-RDI: flow respiratory disturbance index. 


\begin{tabular}{|c|c|c|c|c|}
\hline \multirow[t]{2}{*}{ TABLE 2} & \multicolumn{4}{|c|}{$\begin{array}{l}\text { Agreement between the in-laboratory flow } \\
\text { respiratory disturbance index (RDI) and apnoea/ } \\
\text { hypopnoea index (AHI) }\end{array}$} \\
\hline & & FNH & $\mathrm{TCH}$ & All \\
\hline \multicolumn{2}{|l|}{ Subjects } & 50 & 50 & 100 \\
\hline \multicolumn{2}{|c|}{$\begin{array}{l}\text { Correlation between AHI } \\
\text { and flow-RDI r }\end{array}$} & 0.95 & 0.93 & $0.94(0.91-0.96)$ \\
\hline \multicolumn{2}{|c|}{$\begin{array}{l}\text { Difference between AHI } \\
\text { and flow-RDI }\end{array}$} & $-8.2 \pm 9.1$ & $-10.8 \pm 12.0$ & $-9.5 \pm 10.7$ \\
\hline \multicolumn{5}{|c|}{ Diagnostic ability of flow-RDI } \\
\hline \multicolumn{5}{|c|}{$\mathrm{AHI}>5$ events $\cdot \mathrm{h}^{-1}$} \\
\hline \multicolumn{2}{|c|}{ Sensitivity } & 0.96 & 0.96 & $0.96(0.91-1.00)$ \\
\hline \multicolumn{2}{|c|}{ Specificity } & 0.83 & 0.80 & $0.82(0.59-1.00)$ \\
\hline \multicolumn{2}{|c|}{ P-LR } & 5.7 & 4.8 & $5.3(2.4-11.7)$ \\
\hline \multicolumn{2}{|l|}{ N-LR } & 0.05 & 0.06 & $0.05(0.03-0.09)$ \\
\hline \multicolumn{2}{|l|}{ AUC } & 0.94 & 0.95 & $0.95(0.90-0.99)$ \\
\hline \multicolumn{5}{|c|}{$\mathrm{AHI}>15$ events $\cdot h^{-1}$} \\
\hline \multicolumn{2}{|c|}{ Sensitivity } & 0.90 & 0.91 & $0.91(0.84-0.98)$ \\
\hline \multicolumn{2}{|c|}{ Specificity } & 0.90 & 0.73 & $0.82(0.70-0.95)$ \\
\hline \multicolumn{2}{|c|}{ P-LR } & 8.6 & 3.4 & $5.2(3.9-6.8)$ \\
\hline \multicolumn{2}{|l|}{ N-LR } & 0.11 & 0.12 & $0.11(0.08-0.15)$ \\
\hline \multicolumn{2}{|l|}{ AUC } & 0.97 & 0.94 & $0.96(0.92-0.99)$ \\
\hline \multicolumn{5}{|c|}{$A H I>30$ events $\cdot h^{-1}$} \\
\hline \multicolumn{2}{|c|}{ Sensitivity } & 0.83 & 0.93 & $0.89(0.80-0.97)$ \\
\hline \multicolumn{2}{|c|}{ Specificity } & 0.92 & 1.00 & $0.96(0.90-1.00)$ \\
\hline \multicolumn{2}{|c|}{ P-LR } & 10.8 & NA & $21.2(8.3-54.6)$ \\
\hline \multicolumn{2}{|l|}{ N-LR } & 0.18 & 0.07 & $0.12(0.09-0.16)$ \\
\hline \multicolumn{2}{|l|}{ AUC } & 0.96 & 0.99 & $0.98(0.94-1.00)$ \\
\hline
\end{tabular}

Data are presented as $n$ or mean \pm SD, unless otherwise stated. $95 \%$ confidence intervals are given in parentheses. FNH: Fukuoka National Hosiptal, Fukuoka, Japan; TCH: Tenri City Hospital, Tenri, Japan; r: Pearson's correlation coefficient; P-LR: positive likelihood ratio; N-LR: negative likelihood ratio; AUC: area under the receiver-operating characteristic curve; NA: not available.

\section{Reproducibility of the flow-RDI}

The intraclass correlation coefficients of the flow-RDI between the three nights were $0.92,0.91$ and 0.90 , for home night 1 versus home night 2 , home night 1 versus hospital night and home night 2 versus hospital night, respectively, in $\mathrm{FNH}$, whereas in TCH they were $0.91,0.86$ and 0.88 , respectively, and for all subjects were $0.92,0.88$ and 0.89 , respectively.

\section{Agreement between the $A H I$ and flow-RDI}

The correlation coefficient between the AHI and flow-RDI at hospital night was 0.94 (95\% confidence interval (CI) $0.91-$ 0.96), while that between the AHI at hospital night and flowRDI at home night 1 was 0.84 (CI $0.77-0.89$ ), for combined data (table 2). The Bland and Altman analysis showed that the mean difference between the flow-RDI and the AHI at hospital night was -9.5 (95\% agreement limit -30.4-11.4; fig. 3a). The relatively wide difference is thought to be largely due to the difference in the denominator (examination time versus sleep time), because the difference between the flow-RDI and apnoea/hypopnoea number per hour of examination was far narrower (mean -1.2; 95\% agreement limit -12.9-10.6; fig. 3b).
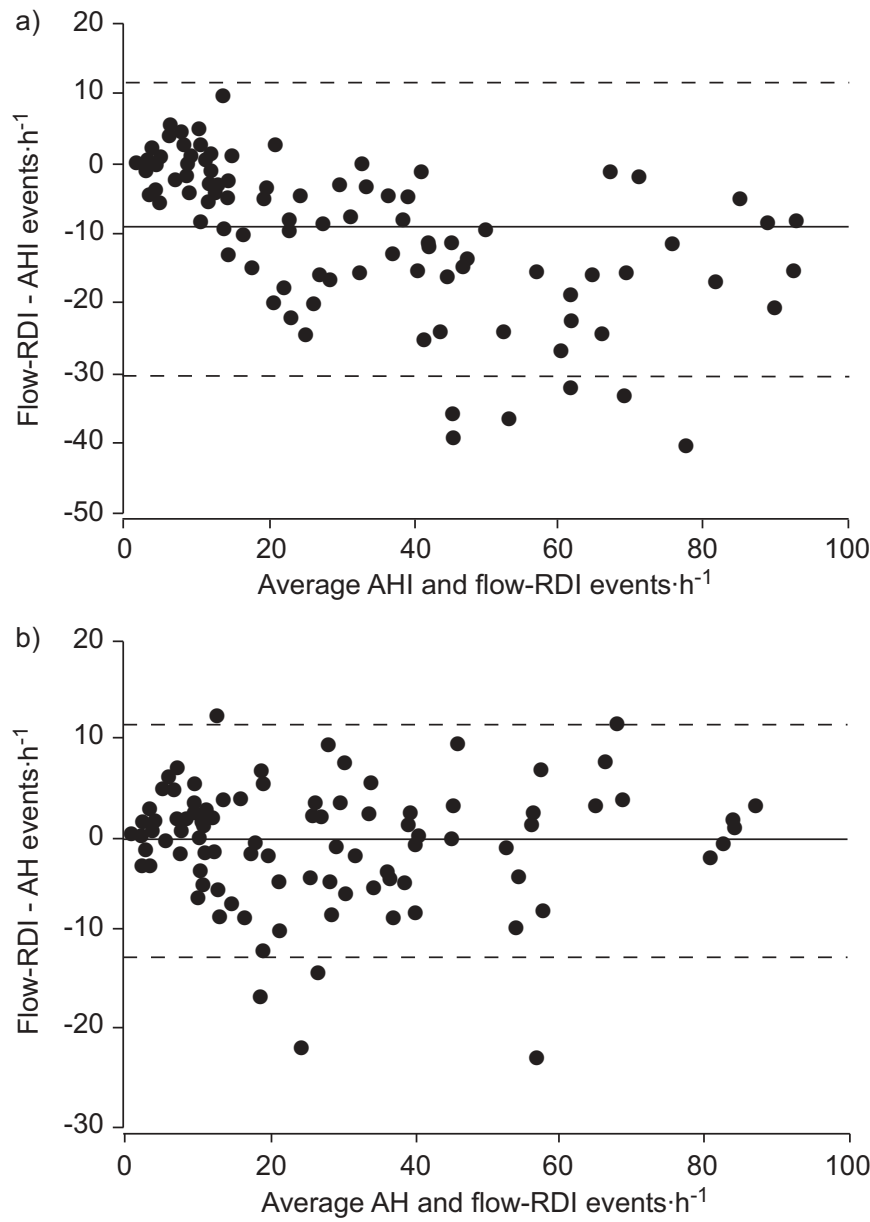

FIGURE 3. Bland and Altman plots. Difference between the flow-respiratory disturbance index (flow-RDI) determined by the in-laboratory SOMNIE and apnoea/ hypopnoea index (AHI) determined by the simultaneous polysomnography was plotted against the average value of the two methods. a) Ordinary AHI (per hour of sleep) was used; b) apnoea/hypopnoea number per hour of recording (AH) was used instead. __ : mean difference; - - - - -: 95\% limit of agreement.

\section{Diagnostic ability of the flow-RDI}

The diagnostic sensitivity and specificity values of the flowRDI for the identification of patients with three different AHI thresholds $\left(\geqslant 5, \geqslant 15\right.$ and $\geqslant 30$ events. $\left.\mathrm{h}^{-1}\right)$ ranged $0.89-0.96$ and $0.82-0.96$, respectively, for the concurrent study with PSG (hospital night). The negative likelihood ratio ranged 0.05-0.12. The overall diagnostic ability, as expressed by the area under the receiver operating characteristic curve, was $0.95-0.98$.

\section{Comparison with oximetry analysis}

The correlation coefficient between the ODI and AHI was 0.95 and the mean difference between the ODI and the AHI was -9.3 (95\% agreement limit -29.2-10.6); these were equivalent to those between the flow-RDI and AHI. However, the correlation between the ODI and AHI was lower in normal weight subjects than that in overweight subjects (fig. 4). In contrast, the correlation between the flow-RDI and AHI was not affected by the body habitus. In addition, in the subjects with AHI $<30$ events $\mathrm{h}^{-1}$, the difference between the flow-RDI and AHI was narrower than that between the ODI and AHI (fig. 5). 

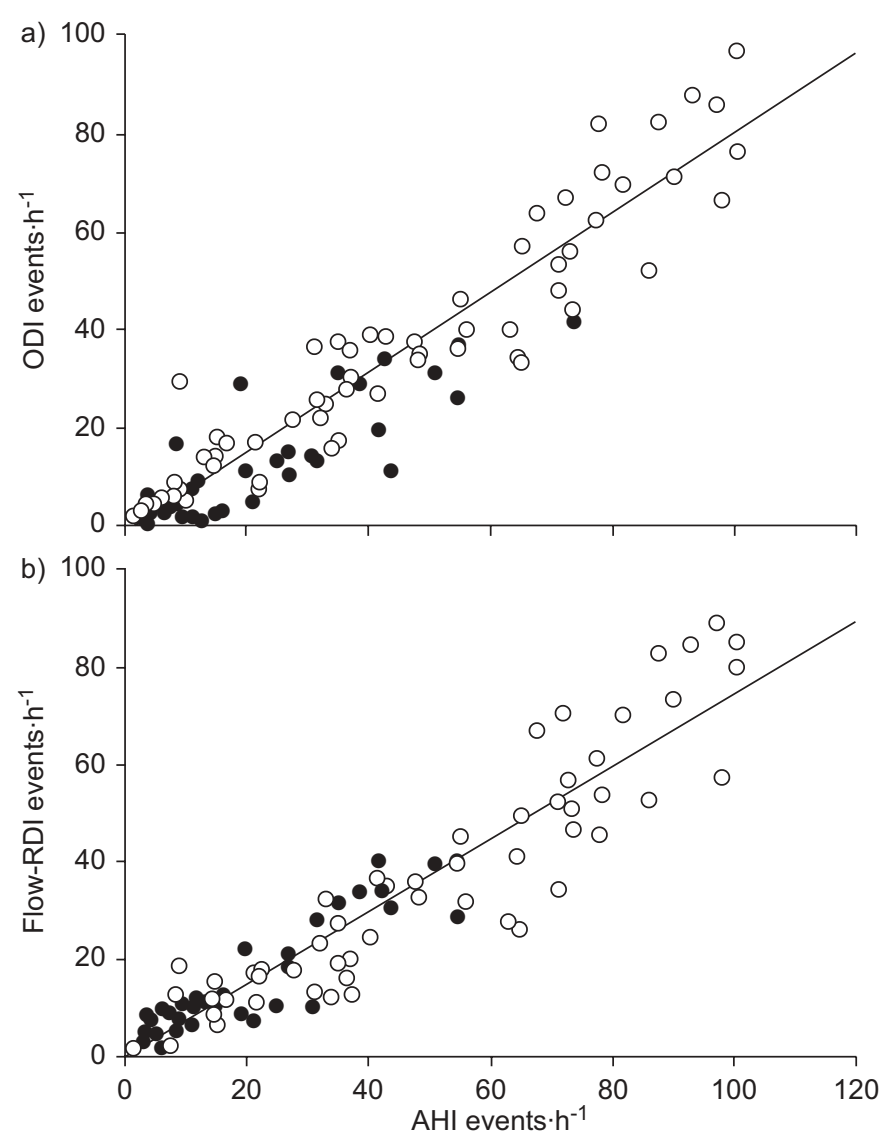

FIGURE 4. The correlation between a) the apnoea/hypopnoea index (AHI) and oxygen desaturation index (ODI) was similar to that b) between the $\mathrm{AHI}$ and flow respiratory disturbance index (flow-RDI) as a whole $(r=0.94$ versus $r=0.94)$. For the normal weight subjects $(\bullet)$ the correlation between the AHI and ODI was poorer than between the $\mathrm{AHI}$ and flow-RDI $(r=0.87$ versus $r=0.93)$. For the overweight subjects $(O)$ the correlations were equivalent $(r=0.94$ versus $r=0.94)$. Regression lines were as follows a) $y=0.8 \times-2$; b) $y=0.74 \times-0.1$.

\section{Evaluation of flow-RDI in low severity subgroups}

Three subgroups of females, normal weight subjects and subjects with AHI $<15$ events $\cdot h^{-1}$ were used for this analysis (table 3). The AHI tended to be lower in the three subgroups. The difference between the flow-RDI at hospital night and the AHI was narrower in all subgroup subjects than in the subjects taken as a whole. The diagnostic ability of the flow-RDI in each subgroup was almost comparable to that of all subjects taken together.

\section{Utility of the subjective sleep log on portable monitoring}

The correlation coefficient between the AHI at hospital night and the flow-RDI at home night 1 was 0.78 and 0.79 before and after excluding subjective waking time, respectively. The mean \pm SD difference between the AHI and flow-RDI was $-11.7 \pm 18.4$ both for before and after excluding subjective waking time. The correlation coefficients between the AHI and the concurrent flow-RDI at hospital night were 0.94 and 0.97 before and after excluding subjective waking time, respectively. The mean \pm SD differences between the AHI and flow-RDI were $-10.3 \pm 11.6$ and $-8.9 \pm 9.2$ before and after excluding subjective
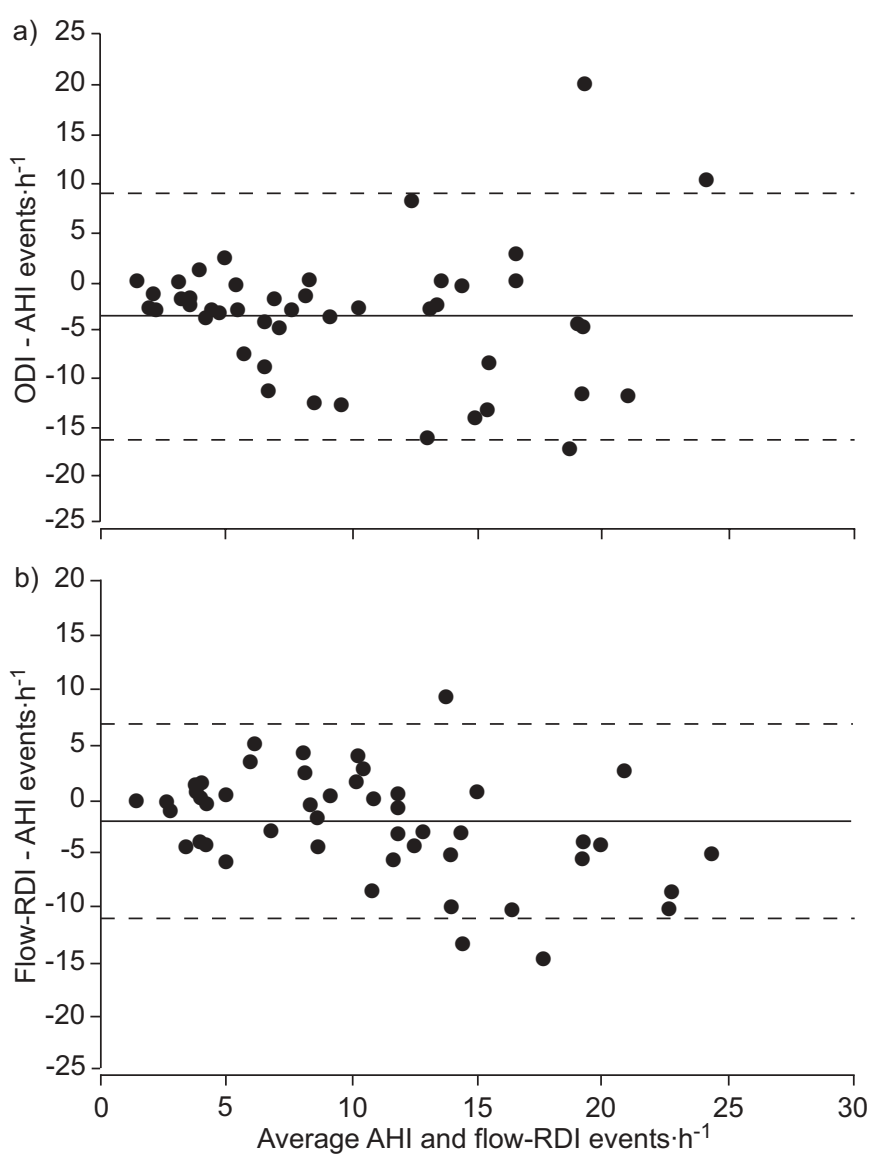

FIGURE 5. Bland and Altman plots in subjects with apnoea/hypopnoea index $(\mathrm{AHI})<30$ events $\cdot \mathrm{h}^{-1}$. a) Difference between the oxygen desaturation index (ODI) and $\mathrm{AHI}$. b) Difference between the flow respiratory disturbance index (flow-RDI) and $\mathrm{AHI}$.

waking time, respectively. The correlation coefficient between subjective sleep efficiency as assessed by the sleep log and actual sleep efficiency by PSG was $0.41(n=48)$.

\section{Event-by-event analysis}

The analysis of event-by-event agreement showed that the SOMNIE automated and manual analysis detected 79 and $74 \%$ respectively, of apnoea and hypopnoea events detected by the full PSG analysis. The ratio of number of false-positive events to that of total events detected by the SOMNIE was $9 \%$ and $7 \%$ for automated and manual analysis, respectively. The PSA between the PSG analysis and the SOMNIE analysis was 0.84, while that between the PSG analysis and the SOMNIE manual analysis was 0.83 . The PSA between the SOMNIE automated and manual analyses was 0.92 .

\section{Result of home monitoring and CPAP use after $1 \mathrm{yr}$}

CPAP treatment was prescribed for 42 patients, out of which 32 patients continued to use it for $\geqslant 1 \mathrm{yr}$. The relationship between the SDB severity by the SOMNIE home night 1 data and continual CPAP use was as follows: normal, 14 patients, CPAP use 0; mild SDB, 22 patients, CPAP use 1; moderate SDB, 21 patients, CPAP use 3; and severe SDB, 43 patients, CPAP use 28 
TABLE 3 Evaluation of in-laboratory flow respiratory disturbance index (flow-RDI) in subgroups

\begin{tabular}{|c|c|c|c|c|}
\hline & All & Female & $\mathrm{BMI}<25 \mathrm{~kg} \cdot \mathrm{m}^{-2}$ & AHI $<15$ events $\cdot h^{-1}$ \\
\hline Subjects $n$ & 100 & 20 & 36 & 34 \\
\hline Correlation between $\mathrm{AHI}$ and flow-RDI $r$ & 0.94 & 0.90 & 0.93 & 0.68 \\
\hline Difference between $\mathrm{AHI}$ and flow-RDI & $-9.5 \pm 10.7$ & $-5.7 \pm 10.2$ & $-5.1 \pm 8.0$ & $-0.4 \pm 3.3$ \\
\hline \multicolumn{5}{|l|}{ Diagnostic ability of flow-RDI } \\
\hline Specificity & 0.82 & 0.67 & 0.71 & 0.82 \\
\hline AUC & 0.95 & 0.88 & 0.94 & 0.81 \\
\hline \multicolumn{5}{|l|}{ AHI $>15$ events $\cdot h^{-1}$} \\
\hline Sensitivity & 0.91 & 0.75 & 0.78 & NA \\
\hline Specificity & 0.82 & 0.83 & 0.89 & NA \\
\hline AUC & 0.98 & 1.00 & 0.97 & NA \\
\hline
\end{tabular}

Data are presented as $n$, mean \pm SD or median (interquartile range), unless otherwise stated. BMI: body mass index; AHI: apnoea/hypopnoea index (median and interquartile range); r: Pearson's correlation coefficient; AUC: area under the receiver-operating characteristic curve; NA: not available.

\section{DISCUSSION}

The single-channel portable monitor used in the present study provided the flow-RDI, which had a relatively high agreement with the AHI assessed with the concurrent PSG recording. The event-by-event agreement between the two methods was also good. The flow-RDI was reproducible for 2 nights at home. There was no recording failure for the home monitoring. These results suggest the portable monitor with the automatic analysis program is feasible in ambulatory screening for SDB.

The flow-RDI tended to be lower than the concurrent PSGderived AHI value. This difference is considered to be predominantly due to the fact that the denominator of flowRDI was recording time while that of the AHI was sleep time, because there was very little difference between the flow-RDI and the apnoea/hypopnoea number per hour of recording. To overcome the weakness of flow-RDI, cut-off values for the flow-RDI were used to detect SDB, and as a result, high diagnostic sensitivity was obtained. In addition, the subjective sleep log was utilised to exclude waking time from the record for analysis. The agreement between the flow-RDI and concurrent AHI was only slightly better after excluding subjective sleep time than before excluding it. However, this procedure had no effect at all on the agreement between the flow-RDI at home and the AHI at hospital. Therefore, the effect of waking time during home monitoring is far more trivial compared with the night-to-night variability of SDB severity.

There have been three single-channel airflow monitors for which data about agreement with simultaneously recorded PSG have been published in the literature. Two monitors utilise a nasal pressure sensor $[9,10]$, and the other uses a thermistor [8]. The correlation coefficient between the portable monitor-derived index and PSG-derived AHI was 0.80 [10] and 0.89 [9] for the two monitors utilising the nasal pressure sensor, whereas it was 0.73 for the monitor using the thermistor [8]. The diagnostic ability expressed as the area under the receiver operating characteristic curve for detection of the case with an AHI of $\geqslant 5$ events $\cdot h^{-1}$ was 0.89 [10] and 0.86 [9] for the two monitors utilising the nasal pressure sensor. The present study showed the correlation coefficient was 0.94 and the area under the receiver operating characteristic curve was 0.95, demonstrating equivalent or superior agreement with PSG compared with the portable monitors utilising the nasal pressure sensor.

Another modality of single-channel monitoring is oximetry alone. There are many reports about the diagnostic ability of this method. Some studies have shown very good diagnostic ability of oximetry alone $[17,18]$. However, this method is known to have a drawback in that the results are affected by the body habitus of the subject [19]. The present study demonstrated that the agreement of the oximetry-derived index with the AHI was worse in normal weight subjects than in overweight subjects, but this was not the case for the flow-RDI.

The crucial parts of portable monitors for accurate detection of SDB are the airflow sensor and the algorithm to detect breathing events. Conventional thermal sensors are known to have a tendency to overlook hypopnoea events and are considered inadequate for sleep study [20]. The portable monitor in the present study utilised a PVDF film. Recently, BERRY et al. [21] have reported that the PVDF airflow sensor has excellent ability to detect hypopnoea. Regarding the analytical algorithm, the recently developed algorithm for single-channel airflow monitors was used. The present authors have demonstrated that the algorithm was applicable to different types of sensors, including thermocouple, nasal pressure and PVDF sensors, and was relatively immune to noises and change in sensitivity over long recording periods [11]. The high ability of the sensor and the robustness of the algorithm could be the 
reasons for the observed high accuracy and reproducibility of the portable monitor used in the present study.

Several limitations in the current study must be addressed. The subjects studied were patients referred for suspected sleep apnoea and not subjects from the general population. Therefore, the present findings may not be applicable to the general population. The subjects in the present study might have been better motivated to perform the home monitoring than members of the general population, which may be the reason that no recording failure was observed. Furthermore, the subjects had different characteristics from a cross section of the general population, being predominantly male, and having a higher than average BMI. Therefore, the diagnostic ability of the flowRDI was examined in subgroups of female subjects and normal weight subjects. The results showed the diagnostic ability in such subgroups was similar to that of all of the subjects taken together. Another problem is that the subjects' average AHI was far greater than that of SDB subjects in the general population. This fact could be a reason for the high diagnostic sensitivity of the flow-RDI in the present study. To exclude this possibility, the diagnostic ability of the flow-RDI in subjects with AHI $<15$ events $\cdot h^{-1}$ was also examined, as a result of which the flowRDI was shown to have a relatively high diagnostic ability, even in this low severity subgroup. Although an exact validation in a cross section of the general population remains to be performed, these facts might suggest that the portable monitor can be used for screening of SDB from a variety of populations. The other important problem is the effect of sleep efficiency. The mean sleep efficiency of the subjects in the present study was relatively high. If low sleep efficiency subjects (e.g. patients with insomnia) are tested, the agreement between the flow-RDI and AHI could be quite different.

Finally, the issue of clinical utility of the device should be addressed. The most possible use of the single-channel monitor is thought to be in case finding in primary care practices. Many of subjects from the present study were referred for the symptom of habitual snoring without overt sleepiness, which can be underestimated by the subjects suffering from long-standing sleep disturbance [22]. In a study in primary care settings, $37.5 \%$ of the patients were identified as being in a high-risk group of sleep apnoea syndrome [23]. It seems somewhat difficult to perform PSG in all of such patients. Thus, ambulatory screening using a portable monitor in this group is thought to be a practical way to determine candidates for PSG. In the present study, 31 out of 32 patients continuing to use CPAP for $1 \mathrm{yr}$ after the diagnosis had the home monitoring data corresponding to moderate-to-severe SDB, which indicates that the portable monitor could be used to screen for candidates for CPAP therapy. Home monitoring also has the possible advantage that subjects can undergo the screening test in their usual sleep environment because sleep position and alcohol drinking can affect the SDB severity of a subject [24]. It is also very important to recognise that the singlechannel monitor does not evaluate sleep and it has no ability to detect sleep disorders other than SDB. Therefore, patients with a negative result and unexplained severe sleepiness should be referred to sleep specialists.

In conclusion, the single-channel airflow monitor is easily applicable at home and relatively accurate. These advantages are thought to be due to both the high-performance sensor (polyvinylidene fluoride) and the robust analytical algorithm.
The present authors believe this method can be applicable in screening for sleep-disordered breathing in primary care practices.

\section{REFERENCES}

1 Young T, Palta M, Dempsey J, Skatrud J, Weber S, Badr S. The occurrence of sleep-disordered breathing among middle-aged adults. N Engl J Med 1993; 328: 1230-1235.

2 McNicholas WT, Bonsignore MR, Management Committee of EU COST ACTION B26., Sleep apnoea as an independent risk factor for cardiovascular disease: current evidence, basic mechanisms and research priorities. Eur Respir J 2007; 29: 156-178.

3 A joint project sponsored by the American Academy of Sleep Medicine, the American Thoracic Society, and the American College of Chest Physicians. Practice parameters for the use of portable monitoring devices in the investigation of suspected obstructive sleep apnea in adults. Sleep 2003; 26: 907-913.

4 Verse T, Pirsig W, Junge-Hülsing B, Kroker B. Validation of the POLY-MESAM seven-channel ambulatory recording unit. Chest 2000; 117: 1613-1618.

5 Parra O, García-Esclasans N, Montserrat JM, et al. Should patients with sleep apnoea/hypopnoea syndrome be diagnosed and managed on the basis of home sleep studies? Eur Respir J 1997; 10: 1720-1724.

6 Dingli K, Coleman EL, Vennelle M, et al. Evaluation of a portable device for diagnosing the sleep apnoea/hypopnoea syndrome. Eur Respir J 2003; 21: 253-259.

7 Golpe R, Jiménez A, Carpizo R. Home sleep studies in the assessment of sleep apnea/hypopnea syndrome. Chest 2002; 122: 1156-1161.

8 Shochat T, Hadas N, Kerkhofs M, et al. The SleepStrip: an apnoea screener for the early detection of sleep apnoea syndrome. Eur Respir J 2002; 19: 121-126.

9 Erman MK, Stewart D, Einhorn D, Gordon N, Casal E. Validation of the ApneaLink for screening of sleep apnea: a novel and simple single-channel recording device. J Clin Sleep Med 2007; 3: 387-392.

10 de Almeida FR, Ayas NT, Otsuka R, et al. Nasal pressure recordings to detect obstructive sleep apnea. Sleep Breath 2006; 10: 62-69.

11 Nakano H, Tanigawa T, Furukawa T, Nishima S. Automatic detection of sleep-disordered breathing from a singlechannel airflow record. Eur Respir J 2007; 29: 728-736.

12 Rechtschaffen A, Kales A. A Manual of Standardized Terminology, Techniques and Scoring Systems for Sleep Stages of Human Subjects. National Institute of Health, Publ. No.204. Washington, Public Health Service, Government Printing Office, 1968.

13 Kushida CA, Littner MR, Morgenthaler T, et al. Practice parameters for the indications for polysomnography and related procedures: an update for 2005. Sleep 2005; 28: 499-521.

14 Bonnet M, Carley D, Carskadon M, et al. EEG arousals: scoring rules and examples: a preliminary report from the Sleep Disorders Atlas Task Force of the American Sleep Disorders Association. Sleep 1992; 15: 173-184.

15 Bland JM, Altman DG. Statistical methods assessing agreement between two methods of clinical measurement. Lancet 1986; 1: 307-310. 
16 Fleiss JL. Statistical Methods for Rates and Proportions. New York, John Wiley \& Sons, 2003; pp. 599-608.

17 Vázquez JC, Tsai WH, Flemons WW, et al. Automated analysis of digital oximetry in the diagnosis of obstructive sleep apnea. Thorax 2000; 55: 302-307.

18 Gurubhagavatula I, Maislin G, Pack A. An algorithm to stratify sleep apnea risk in a sleep disorders clinic population. Am J Respir Crit Care Med 2001; 164: 1904-1909.

19 Nakano H, Ikeda T, Hayashi M, et al. Effect of body mass index on overnight oximetry for the diagnosis of sleep apnea. Respir Med 2004; 98: 421-427.

20 Farre R, Montserrat JM, Rotger M, Ballester E, Navajas D. Accuracy of thermistors and thermocouples as flowmeasuring devices for detecting hypopnoeas. Eur Respir J 1998; 11: 179-182.
21 Berry RB, Koch GL, Trautz S, Wagner MH. Comparison of respiratory event detection by a polyvinylidene fluoride film airflow sensor and a pneumotachograph in sleep apnea patients. Chest 2005; 128: 1331-1338.

22 Hans PA, Van Dongen HP, Maislin G, Mullington JM, David F, Dinges DF. The cumulative cost of additional wakefulness: dose-response effects on neurobehavioral functions and sleep physiology from chronic sleep restriction and total sleep deprivation. Sleep 2003; 26: 117-126.

23 Netzer NC, Stoohs RA, Netzer CM, Clark K, Strohl KP. Using the Berlin questionnaire to identify patients at risk for the sleep apnea syndrome. Ann Intern Med 1999; 131: 485-491.

24 Tanigawa T, Tachibana N, Yamagishi K, et al. Usual alcohol consumption and arterial oxygen desaturation during sleep. JAMA 2004; 292: 923-925. 